\title{
BMJ Open Prognostic significance of dipstick proteinuria in heart failure with preserved ejection fraction: insight from the PURSUIT-HFpEF registry
}

\author{
Bolrathanak Oeun, ${ }^{1}$ Shungo Hikoso (D) , ${ }^{1}$ Daisaku Nakatani, ${ }^{1}$ Hiroya Mizuno, ${ }^{1}$ \\ Shinichiro Suna, ${ }^{1}$ Tetsuhisa Kitamura, ${ }^{2}$ Katsuki Okada, ${ }^{1}$ Tomoharu Dohi, ${ }^{1}$ \\ Yohei Sotomi (D) , ${ }^{1}$ Hirota Kida, ${ }^{1}$ Akihiro Sunaga, ${ }^{1}$ Taiki Sato, ${ }^{1}$ Akito Nakagawa, ${ }^{3,4}$ \\ Yusuke Nakagawa, ${ }^{5}$ Takaharu Hayashi, ${ }^{6}$ Masamichi Yano, ${ }^{7}$ Shunsuke Tamaki, ${ }^{8}$ \\ Yoshio Yasumura, ${ }^{3}$ Takahisa Yamada, ${ }^{8}$ Yasushi Sakata (1) , ${ }^{1}$ The OCVC-Heart \\ Failure Investigators
}

To cite: Oeun B, Hikoso S, Nakatani D, et al. Prognostic significance of dipstick proteinuria in heart failure with preserved ejection fraction: insight from the PURSUITHFpEF registry. BMJ Open 2021;11:e049371. doi:10.1136/ bmjopen-2021-049371

- Prepublication history and additional supplemental material for this paper are available online. To view these files, please visit the journal online (http://dx.doi.org/10.1136/ bmjopen-2021-049371)

Received 24 January 2021 Accepted 02 August 2021

\section{Check for updates}

(c) Author(s) (or their employer(s)) 2021. Re-use permitted under CC BY-NC. No commercial re-use. See rights and permissions. Published by BMJ.

For numbered affiliations see end of article.

\section{Correspondence to}

Dr Shungo Hikoso;

hikoso@cardiology.med.osakau.ac.jp

\section{ABSTRACT}

Objective The semiquantitative urine dipstick test is a simple and convenient method that is available in the smallest community-based healthcare clinics. We sought to clarify the prognostic significance of dipstick proteinuria in patients with heart failure (HF) with preserved ejection fraction (HFpEF).

Design A Prospective mUticente $R$ obServational st $U d y$ of pat/enTs with Heart Failure with preserved Ejection Fraction (PURSUIT-HFpEF) registry.

Participants and setting We assessed 851 dischargedalive patients in the PURSUIT-HFpEF registry who were initially hospitalised due to an acute decompensated HFpEF (EF $\geq 50 \%$ ) and elevated N-terminal-pro-brain natriuretic peptide ( $\geq 400 \mathrm{ng} / \mathrm{L}$ ) at Osaka University Hospital and other 30 affiliated hospitals in the Kansai region of Japan. Patients received a urine dipstick test, and were divided into two groups according to the absence or presence of proteinuria. A trace or more of dipstick proteinuria was defined as the presence of proteinuria.

Main outcome measures A composite of cardiac death or HF rehospitalisation.

Results Median age was 83 years and 473 patients $(55.6 \%)$ were female. Five hundred and two patients $(59 \%)$ were proteinuria (-) and 349 patients $(41 \%)$ were proteinuria $(+)$. The composite endpoint and HF rehospitalisation occurred more often in proteinuria $(+)$ individuals than proteinuria (-) individuals (log-rank $p=0.006$ and $p=0.007$, respectively); but cardiac death did not (log-rank $p=0.139)$. Multivariable Cox regression analysis showed that the presence of proteinuria was associated with the composite endpoint (HR: $1.47,95 \% \mathrm{Cl}$ 1.07 to 2.01, $\mathrm{p}=0.016$ ), and HF rehospitalisation (HR: 1.48 , $95 \% \mathrm{Cl} 1.07$ to $2.05, \mathrm{p}=0.020$ ), but not with cardiac death (HR: $1.52,95 \% \mathrm{Cl} 0.83$ to $2.76, \mathrm{p}=0.172$ ).

Conclusions Dipstick proteinuria may be a prognostic marker in patients with HFpEF. Evaluation of proteinuria by a urine dipstick test may be a simple but useful method for risk stratification in HFpEF.

UMIN-CTR ID UMIN000021831.

\section{Strengths and limitations of this study}

- The large prospective multicenter observational study with essential information to clarify the association between dipstick proteinuria and prognosis in real-world patients with heart failure with preserved ejection fraction (HFpEF)

- Enough sample size and event number enable the detailed adjustment for essential confounding factors in multivariable Cox regression analysis.

- Detailed subgroup analyses are useful to estimate the impact of proteinuria in patients with specific characteristics.

- The East Asian elderly HFpEF population in this study may limit the generalisability of the results to other populations.

- Further study is needed to compare the prognostic utility of dipstick proteinuria with that of urinary albumin-to-creatinine ratio.

\section{BACKGROUND}

Heart failure (HF) with preserved ejection fraction (HFpEF) is a major public health issue worldwide, especially in ageing societies such as Japan. It is associated with poor clinical outcomes. ${ }^{12}$ Although a growing body of research has been conducted to understand the complex pathophysiologic abnormalities of HFpEF, no optimal and effective treatment has yet been established. ${ }^{34}$

Chronic kidney disease (CKD) is one of the most common comorbidities and frequently associated with patients with HFpEF. ${ }^{5-7}$ It is closely related to HFpEF development, and worsens its prognosis through different pathways including volume expansion, systemic inflammation, arterial stiffening and hypertension, neurohormonal changes and 
worsening cardiac mechanics. ${ }^{68}$ Proteinuria is not only a well-known marker of CKD but also reflects endothelial microvascular dysfunction and systemic inflammation, ${ }^{10}$ and is associated with cardiac remodelling and cardiovascular events. ${ }^{51-13}$ Proteinuria may appear earlier than the deterioration of renal function detected by the elevation of serum creatinine. ${ }^{1415}$ Several methods are used to detect proteinuria in random urine samples, including the urine dipstick test and the urinary albumin-tocreatinine ratio (UACR). Although UACR has advantages over the urine dipstick test in sensitivity, quantification and accuracy, and is reported to be a prognostic indicator in patients with HFpEF, ${ }^{11}{ }^{13}$ measurement of UACR requires expensive equipment and may not be practical in daily practice. It was not performed routinely on patients in this registry. Previous studies assessing renal function by both estimated glomerular filtration rate (eGFR) and proteinuria demonstrated that proteinuria in addition to eGFR was useful for risk stratification and prognosis in $\mathrm{HF}^{716}$ Although the predictive value of proteinuria has been previously reported, ${ }^{712} 13$ the prognostic impact of isolated proteinuria detected by the urine dipstick test on clinical outcomes in patients with HFpEF remains to be elucidated.

Here, we investigated the prognostic significance of dipstick proteinuria on adverse outcomes in patients with HFpEF using the database of the Prospective mUlticente $R$ obServational st $U$ dy of pat $J e n T$ s with Heart Failure with preserved Ejection Fraction (PURSUIT-HFpEF) registry.

\section{METHODS}

The PURSUIT-HFpEF registry is being conducted by Osaka University Hospital in collaboration with 30 local hospitals in the Kansai region of Japan. ${ }^{17}$ This study collects and records extensive clinical, echocardiographic, therapeutic and prognostic data of patients hospitalised for acute decompensated HFpEF, for use in clarifying clinical questions and explaining the pathophysiology of this entity. This registry enrolled patients with HFpEF admitted due to decompensated HF diagnosed using the Framingham heart failure diagnostic criteria. ${ }^{18}$ HFpEF was diagnosed when a case satisfied the following criteria: (1) left ventricular ejection fraction $\geq 50 \%$ as measured using the modified Simpson method or Teichholz method by transthoracic echocardiography (TTE) and (2) N-terminal pro-B-type natriuretic peptide (NT-pro-BNP) $\geq 400 \mathrm{ng} / \mathrm{L}$ or $\mathrm{BNP} \geq 100 \mathrm{ng} / \mathrm{L}$ on admission. We excluded patients aged $<20$ years, those with severe valvular diseases (aortic stenosis, aortic regurgitation, mitral stenosis or mitral regurgitation) due to structural changes in valves detected by TTE on admission, those with acute coronary syndrome on admission, those with a life expectancy of $<6$ months due to noncardiac diseases, those with a history of heart transplantation and those considered ineligible for the study by the attending physicians.

\section{Data collection}

Investigational cardiologists and research nurses at the participating institutions are encouraged to enrol consecutive patients in the study and record necessary data on admission, at discharge and at follow-up visits into the electric medical record systems. Data on demography, medical history and comorbidities were collected on admission. Body mass index (BMI; calculated as weight in kilograms divided by height in $\mathrm{m}^{2}$ ), systolic blood pressure, heart rate, atrial fibrillation (AF), New York Heart Association (NYHA) classification, laboratory and echocardiographic data and medications were obtained at discharge. The cut-off values for diastolic function parameters (mean e' septal and lateral wall, mean E/e', left atrial volume index, tricuspid regurgitation velocity and left ventricular mass index) were based on the clinical guideline released from the European Society of Cardiology in 2016. ${ }^{3}$ The recorded data are then transferred to the data centre of Osaka University Hospital via a secure internet connection for processing and analysis.

\section{Study subjects}

Of the data of 1024 cases enrolled in the registry between May 2016 and July 2020, we excluded 16 cases of in-hospital death, 9 cases of cardiac amyloidosis and sarcoidosis, 6 cases of pulmonary arterial hypertension and 142 cases without urinary protein measurements (10 cases of hemodialysis). We excluded cases with cardiac amyloidosis or sarcoidosis or pulmonary arterial hypertension from this analysis, as well as cases with severe valvular diseases from the registry because of their markedly different characteristics, specific haemodynamic and pathophysiological features from common HFpEF. Most randomised clinical trials ${ }^{19-21}$ and observational studies ${ }^{22}$ of HFpEF have excluded these cases. Consequently, we analysed 851 discharged-alive cases and classified them into two groups according to the absence (502 cases, $59 \%$ ) or presence (349 cases, $41 \%$ ) of proteinuria (figure 1).

\section{Patient and public involvement}

There was no patient and public involvement in the design, conduct, choice of outcome measures and plans for dissemination of the study results.

\section{Definition of proteinuria and CKD}

A urine dipstick test was performed at discharge. Proteinuria $\geq$ 'trace' by dipstick was defined as positive, which usually means microalbuminuria (UACR $\geq 30 \mathrm{mg} / \mathrm{g}$ ) as described in previous studies. ${ }^{72324}$ CKD was defined as either one of the following conditions lasting for $>3$ months: (1) findings suggesting kidney damage, that is, abnormal findings in blood or urine tests, imaging studies or pathological evaluations and (2) eGFR $<60 \mathrm{~mL} /$ $\min / 1.73 \mathrm{~m}^{2}{ }^{25}$ For sensitivity analyses, we tried to classify patients using different cut-off points of proteinuria. First, we included patients with proteinuria (-) and 'trace' proteinuria into one group (called 'proteinuria $\leq$ trace'), and compared them with those with proteinuria $\geq 1+$. In 


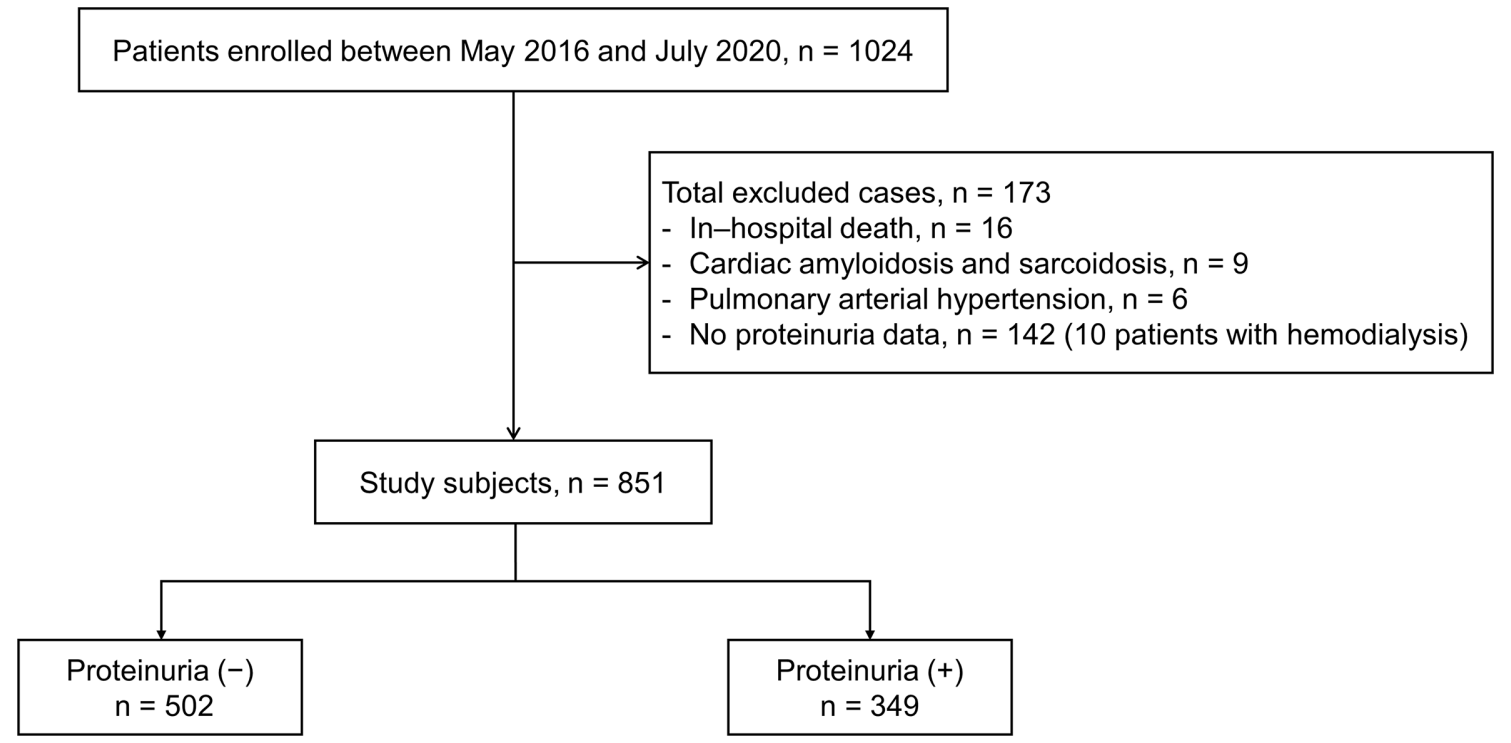

Figure 1 Patient selection and classification.

addition, we classified patients into three groups (proteinuria $(-)$, trace or proteinuria $1+$ and proteinuria $\geq 2+$ ) to explore a linear association between the severity of proteinuria and risk of the composite endpoint.

\section{Study endpoint}

The primary endpoint of this study was a composite of cardiac death or HF rehospitalisation, whereas the secondary endpoint was individual cardiac death or HF rehospitalisation occurring after discharge. Enrolled patients were followed up at outpatient clinics and clinical outcomes data including mortality, detailed causes of death and HF rehospitalisation were collected by direct contact or phone call with patients or relatives or through the National Vital Statistics to ensure a high follow-up rate.

\section{Statistical analysis}

Considering the explorative and observational nature of the current study, no formal sample size was calculated. We have enrolled all consecutive patients who fulfilled the criteria mentioned in the part of 'Study subjects'. Continuous variables were presented as median (IQR: 25\%-75\%), and were compared using a non-parametric Mann-Whitney $\mathrm{U}$ test. Categorical variables were expressed as number (\%), and were compared using the $\chi^{2}$ test. Survival curves of the study cohort stratified by the absence or presence of proteinuria were estimated using the Kaplan-Meier analysis and statistical differences were detected using the log-rank test. Incidence rates of clinical outcomes according to proteinuria status were calculated with the number of events divided by person-years. Univariable and multivariable Cox regression analyses were performed to clarify the independent prognostic impact of proteinuria on the disease outcomes. Major confounding factors considered based on their clinical importance and findings in previous studies, ${ }^{726}$ namely, age; female sex; BMI; hypertension; diabetes mellitus
(DM); previous myocardial infarction (MI); stroke; cancer; heart rate; AF; NYHA classification $\geq \mathrm{III}$; haemoglobin; eGFR; serum albumin; log-transformed NT-pro-BNP; and use of angiotensin-converting enzyme inhibitors (ACEIs) or angiotensin receptor blockers (ARBs) and/or diuretics were added into the multivariable Cox regression model for the composite endpoint. Adjusted covariates were assessed with multicollinearity test and proportional hazard assumption test using Schoenfeld residuals. All covariates showed absence of multicollinearity. All covariates, except serum albumin (Schoenfeld residuals $\mathrm{p}<0.05$ ), satisfied the proportionality test. We dealed with the violation of proportionality using a time-dependent covariate method. ${ }^{27}{ }^{28}$ An interaction term between time and serum albumin was included in the time-dependent multivariable Cox regression models, in addition to serum albumin itself and other fixed covariates. We removed four covariates, namely, stroke, cancer, heart rate and NYHA $\geq$ III from the multivariable Cox regression models for the secondary endpoint to avoid overfitting. In addition, to examine whether the prognostic impact of proteinuria varied in different subgroups of patients, multivariable Cox regression analyses controlling for age and female sex were conducted for subgroups of age $\geq 83$ years (median) versus age $<83$ years, male versus female, DM $(-)$ versus DM $(+)$, previous HF admission $(-)$ versus previous $\mathrm{HF}$ admission $(+), \mathrm{AF}(-)$ versus $\mathrm{AF}$ $(+)$, eGFR $<60 \mathrm{~mL} / \mathrm{min} / 1.73 \mathrm{~m}^{2}$ versus eGFR $\geq 60 \mathrm{~mL} /$ $\mathrm{min} / 1.73 \mathrm{~m}^{2}$, NT-pro-BNP $<1050 \mathrm{ng} / \mathrm{L}$ (median) versus NT-pro-BNP $\geq 1050 \mathrm{ng} / \mathrm{L}$, ACEI/ARBs (-) versus ACEI/ ARBs $(+)$, $\beta$-blocker (BB) (-) versus BB $(+)$ and mineralocorticoid receptor antagonists (MRAs) (-) versus MRAs (+). We selected clinically important subgroups for the management of HFpEF. We evaluated multiplicative interactions between proteinuria and other covariates by introducing the product of proteinuria and a covariate of interest as the interaction terms in the multivariable 
Table 1 Baseline patient characteristics

\begin{tabular}{|c|c|c|c|c|c|}
\hline & Proteinuria (-) & Proteinuria (+) & Total & & \\
\hline & $\mathrm{n}=502$ & $n=349$ & $\mathrm{n}=851$ & Missing & $P$ value \\
\hline Age, years & $83(78,87)$ & $82(76,87)$ & $83(77,87)$ & 0 & 0.073 \\
\hline Female, n (\%) & $300(59.8)$ & $173(49.6)$ & $473(55.6)$ & 0 & 0.003 \\
\hline Body mass index, $\mathrm{kg} / \mathrm{m}^{2}$ & $21.3(18.8,23.9)$ & $22.2(19.3,25.3)$ & $21.5(19.0,24.3)$ & 8 & 0.002 \\
\hline Systolic blood pressure, $\mathrm{mm} \mathrm{Hg}$ & $115(104,126)$ & $124(110,137)$ & $118(106,130)$ & 0 & $<0.001$ \\
\hline Heart rate, beats/min & $70(61,79)$ & $71(62,80)$ & $70(61,80)$ & 0 & 0.506 \\
\hline \multicolumn{6}{|l|}{ Medical history, n (\%) } \\
\hline Previous HF admission & $115(23.4)$ & $92(27.1)$ & 207 (24.9) & 19 & 0.227 \\
\hline Hypertension & $415(82.8)$ & $302(87.0)$ & 717 (84.6) & 3 & 0.096 \\
\hline Diabetes mellitus & $131(26.5)$ & $154(44.1)$ & $285(33.8)$ & 7 & $<0.001$ \\
\hline Dyslipidaemia & $194(39.1)$ & $159(45.7)$ & $353(41.8)$ & 7 & 0.057 \\
\hline Previous myocardial infarction & $27(5.5)$ & $31(9.0)$ & $58(6.9)$ & 16 & 0.047 \\
\hline COPD & $33(6.9)$ & $30(9.0)$ & $63(7.8)$ & 43 & 0.273 \\
\hline Chronic kidney disease & $162(32.5)$ & $171(49.4)$ & $333(39.5)$ & 7 & $<0.001$ \\
\hline Stroke & $79(15.9)$ & $53(15.4)$ & $132(15.7)$ & 10 & 0.848 \\
\hline Cancer & $58(11.8)$ & $41(11.9)$ & $99(11.8)$ & 15 & 0.975 \\
\hline AF at discharge, $\mathrm{n}(\%)$ & $203(40.5)$ & $123(35.2)$ & $326(38.4)$ & 1 & 0.120 \\
\hline NYHA $\geq I I I, n(\%)$ & $38(7.7)$ & $23(6.6)$ & $61(7.3)$ & 12 & 0.547 \\
\hline \multicolumn{6}{|l|}{ Laboratory data at discharge } \\
\hline Haemoglobin, g/L & $115(102,128)$ & $110(98,124)$ & $113(101,126)$ & 1 & 0.020 \\
\hline eGFR, mL/min/1.73 m2 & $45.0(34.4,58.5)$ & $37.4(23.6,51.7)$ & $41.8(30.3,55.4)$ & 12 & $<0.001$ \\
\hline Albumin, g/L & $35(32,38)$ & $33(31,37)$ & $34(31,37)$ & 13 & $<0.001$ \\
\hline $\mathrm{Na}, \mathrm{mEq} / \mathrm{L}$ & $139(137,141)$ & $140(137,142)$ & $139(137,141)$ & 2 & 0.023 \\
\hline $\mathrm{K}, \mathrm{mEq} / \mathrm{L}$ & $4.3(3.9,4.6)$ & $4.3(3.9,4.6)$ & $4.3(3.9,4.6)$ & 1 & 0.309 \\
\hline NT-pro-BNP, ng/L & $891(388,1903)$ & $1290(564,2777)$ & $1050(469,2359)$ & 74 & $<0.001$ \\
\hline $\mathrm{CRP}, \mathrm{mg} / \mathrm{L}$ & $2.2(1.0,6.5)$ & $3.6(1.4,11.2)$ & $2.7(1.1,8.2)$ & 7 & $<0.001$ \\
\hline Fasting glucose, $\mathrm{mmol} / \mathrm{L}$ & $5.27(4.77,6.22)$ & $5.66(5.05,7.05)$ & $5.44(4.88,6.55)$ & 43 & $<0.001$ \\
\hline LVEF (modified Simpson) at discharge, \% & $61.0(56.0,65.8)$ & $61.0(55.0,65.6)$ & $61.0(55.7,65.6)$ & 105 & 0.470 \\
\hline Mean e' septal and lateral wall $<9 \mathrm{~cm} / \mathrm{s}^{*}, \mathrm{n}(\%)$ & $382(85.3)$ & $273(89.8)$ & $655(87.1)$ & 99 & 0.069 \\
\hline Mean E/e' $\geq 13,{ }^{*} n(\%)$ & $181(40.7)$ & $164(54.1)$ & $345(46.1)$ & 103 & $<0.001$ \\
\hline LAVI $>34$ mL $/ \mathrm{m}^{2},{ }^{*} \mathrm{n}(\%)$ & $345(80.4)$ & $237(79.3)$ & $582(79.9)$ & 123 & 0.702 \\
\hline TR velocity $>2.8 \mathrm{~m} / \mathrm{s},{ }^{*} \mathrm{n}(\%)$ & $118(24.3)$ & $108(32.0)$ & $226(27.5)$ & 28 & 0.014 \\
\hline $\begin{array}{l}\text { LVMI } \geq 115 \mathrm{~g} / \mathrm{m}^{2} \text { for } \text { males }^{*} \text { or } \geq 95 \mathrm{~g} / \mathrm{m}^{2} \text { for } \\
\text { females, }{ }^{*} \mathrm{n}(\%)\end{array}$ & 218 (45.5) & $172(52.0)$ & $390(48.1)$ & 41 & 0.071 \\
\hline $\begin{array}{l}\text { Positive at least one index of functional or } \\
\text { structural alterations, } \dagger \mathrm{n}(\%)\end{array}$ & $462(93.7)$ & $326(96.4)$ & $788(94.8)$ & 20 & 0.080 \\
\hline \multicolumn{6}{|l|}{ Medications at discharge, n (\%) } \\
\hline ACEIs/ARBs & $282(56.2)$ & $186(53.3)$ & $468(55)$ & 0 & 0.406 \\
\hline$\beta$-blockers & $275(54.8)$ & $188(53.9)$ & $463(54.4)$ & 0 & 0.793 \\
\hline Diuretics & $408(81.3)$ & $291(83.4)$ & $699(82.1)$ & 0 & 0.430 \\
\hline MRAs & $200(39.8)$ & $131(37.5)$ & 331 (38.9) & 0 & 0.498 \\
\hline
\end{tabular}

Values are presented as number (\%) and median (IQR).

${ }^{*}$ Cut-off values were based on 2016 ESC guidelines for the diagnosis and treatment of acute and chronic heart failure. ${ }^{3}$

†Functional or structural alterations: mean e' septal and lateral wall $<9 \mathrm{~cm} / \mathrm{s}$ or mean E/e' $\geq 13$ or LAVl $>34 \mathrm{~mL} / \mathrm{m}^{2}$ or TR velocity $>2.8 \mathrm{~m} / \mathrm{s}$ or LVMI $\geq 115 \mathrm{~g} / \mathrm{m}^{2}$ for males or $\geq 95 \mathrm{~g} / \mathrm{m}^{2}$ for females.

ACEls, angiotensin-converting enzyme inhibitors; AF, atrial fibrillation; ARBs, angiotensin receptor blockers; COPD, chronic obstructive pulmonary disease; CRP, C reactive protein; eGFR, estimated glomerular filtration rate; HF, heart failure; LAVI, left atrial volume index; LVEF, left ventricular ejection fraction; LVMI, left ventricular mass index; MRAs, mineralocorticoid receptor antagonists; NYHA, New York Heart Association; TR, tricuspid regurgitation. 
A

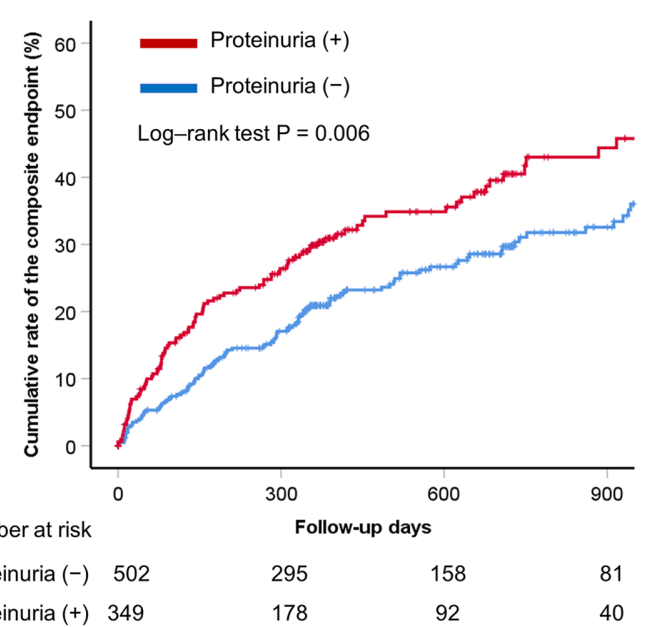

Proteinuria (+) 349

\section{B}

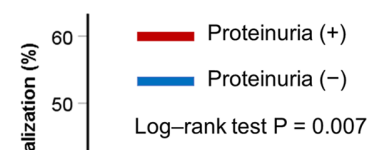

Log-rank test $P=0.007$

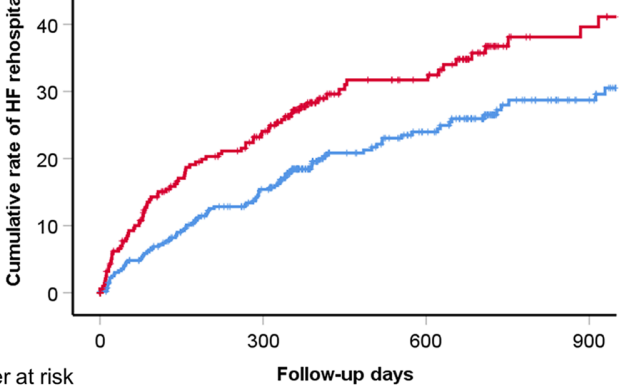

Number at risk

Proteinuria (-) 502

Follow-up days

Proteinuria (+) 349

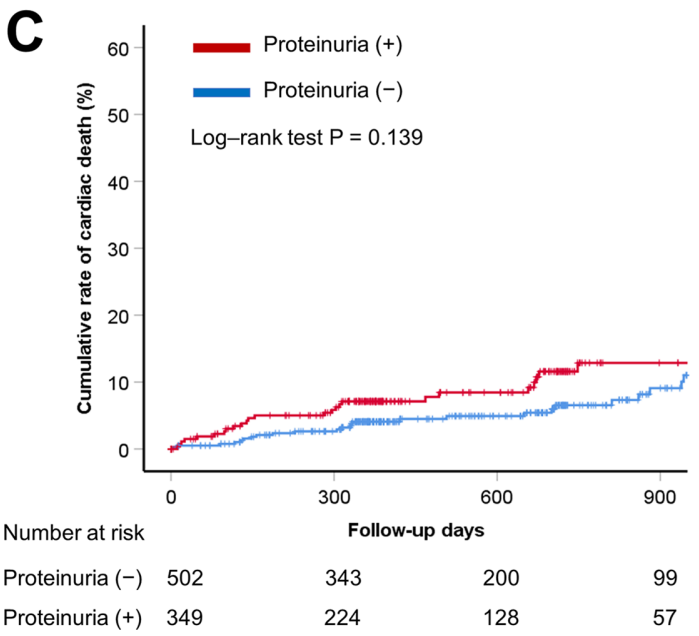

Figure 2 Kaplan-Meier curves according to the absence or presence of proteinuria. (A) Composite endpoint of cardiac death or HF rehospitalisation, (B) HF rehospitalisation and (C) cardiac death. HF, heart failure.

Cox regression models for the composite endpoint in addition to proteinuria and that covariate of interest themselves. A two-sided $\mathrm{p}$ value of $<0.05$ and $\mathrm{p}$ value for interaction tests of $<0.10$ were considered statistically significant. We described the number of missing data in table 1. Listwise deletion was conducted for missing data

\begin{tabular}{|c|c|c|c|c|c|c|}
\hline & Proteinuria & Person-year & Cases & IR per 100 & $\begin{array}{l}\text { Unadjusted } \\
\text { HR }(95 \% \mathrm{Cl})\end{array}$ & $\begin{array}{l}\text { Adjusted } \\
\text { HR }(95 \% \mathrm{Cl})\end{array}$ \\
\hline \multirow{2}{*}{$\begin{array}{l}\text { Composite of cardiac death } \\
\text { or HF rehospitalisation }\end{array}$} & $(-)$ & 577.25 & 114 & 19.7 & & \\
\hline & $(+)$ & 334.20 & 99 & 29.6 & 1.46 (1.11 to 1.91$)$ & 1.47 (1.07 to 2.01$)$ \\
\hline Cardiac death & $(+)$ & 413.56 & 26 & 6.3 & 1.49 (0.88 to 2.55$)$ & $1.52(0.83$ to 2.76$)$ \\
\hline \multirow[t]{2}{*}{ HF rehospitalisation } & $(-)$ & 577.25 & 97 & 16.8 & & \\
\hline & $(+)$ & 334.20 & 87 & 26.0 & 1.49 (1.11 to 1.99$)$ & 1.48 (1.07 to 2.05$)$ \\
\hline
\end{tabular}

Adjusted variables for primary endpoint: age, female sex, body mass index, hypertension, diabetes mellitus, previous myocardial infarction, stroke, cancer, heart rate, atrial fibrillation, NYHA $\geq 1 \mathrm{Il}$, haemoglobin, eGFR, serum albumin (treated as a time-dependent covariate), log-transformed NT-proBNP, use of ACEI/ARBs and diuretics.

Adjusted variables for secondary endpoint: age, female sex, body mass index, hypertension, diabetes mellitus, previous myocardial infarction, atrial fibrillation, haemoglobin, eGFR, serum albumin (treated as a time-dependent covariate), log-transformed NT-pro-BNP, use of ACEI/ARBs and diuretics.

ACEIs, angiotensin-converting enzyme inhibitors; ARBs, angiotensin receptor blockers; eGFR, estimated glomerular filtration rate; HF, heart failure; IR, incidence rate; NT-pro-BNP, N-terminal pro B-type natriuretic peptide; NYHA, New York Heart Association. 
in the multivariable Cox regression models. Any suspicious data were double-checked for accuracy by investigators to mitigate potential bias. All analyses were carried out using IBM SPSS Statistics for Windows V.26.0.

\section{RESULTS}

\section{Baseline characteristics of the study population}

Detailed case characteristics are presented in table 1 . Median age was 83 years, and $55.6 \%$ of the study subjects were female. Briefly, compared with cases without proteinuria, cases with proteinuria were more likely to be male; to have higher BMI, blood pressure, prevalence of diabetes, previous MI, CKD, higher NT-pro-BNP and $\mathrm{C}$ reactive protein (CRP); and to have lower haemoglobin, eGFR and serum albumin. There were no significant differences in the discharge medications between the two groups. Cases with proteinuria had a significantly higher prevalence of high mean $\mathrm{E} / \mathrm{e}^{\prime}$ and high tricuspid regurgitation velocity than those without. Of 851 total study subjects, 788 (94.8\%) had at least one positive criterion for functional or structural alterations as previously recommended. ${ }^{3}$

\section{Clinical outcomes}

During a median follow-up of 344 (18-676) days, 213 cases (25\%) reached the composite endpoint. The composite endpoint and HF rehospitalisation occurred more frequently in cases with proteinuria compared with those without (log-rank $\mathrm{p}=0.006, \mathrm{p}=0.007$, respectively) (figure 2A,B). In contrast, the cardiac death rate was not significantly different between the two groups (log-rank $\mathrm{p}=0.139$ ) (figure 2C). The detailed incidence rates of these clinical events are shown in table 2.

Univariable Cox regression analysis revealed that the presence of proteinuria was associated with the composite endpoint and HF rehospitalisation, but not with cardiac death. Multivariable Cox regression analysis indicated that the presence of proteinuria was independently associated with the composite endpoint and HF rehospitalisation, but not with cardiac death (table 2).

Subgroup analyses demonstrated that the impact of proteinuria on the composite endpoint differed between cases administered and not administered MRAs ( $p$ value for interaction $=0.080$; figure 3 ). In contrast, there were no significant interactions between proteinuria and

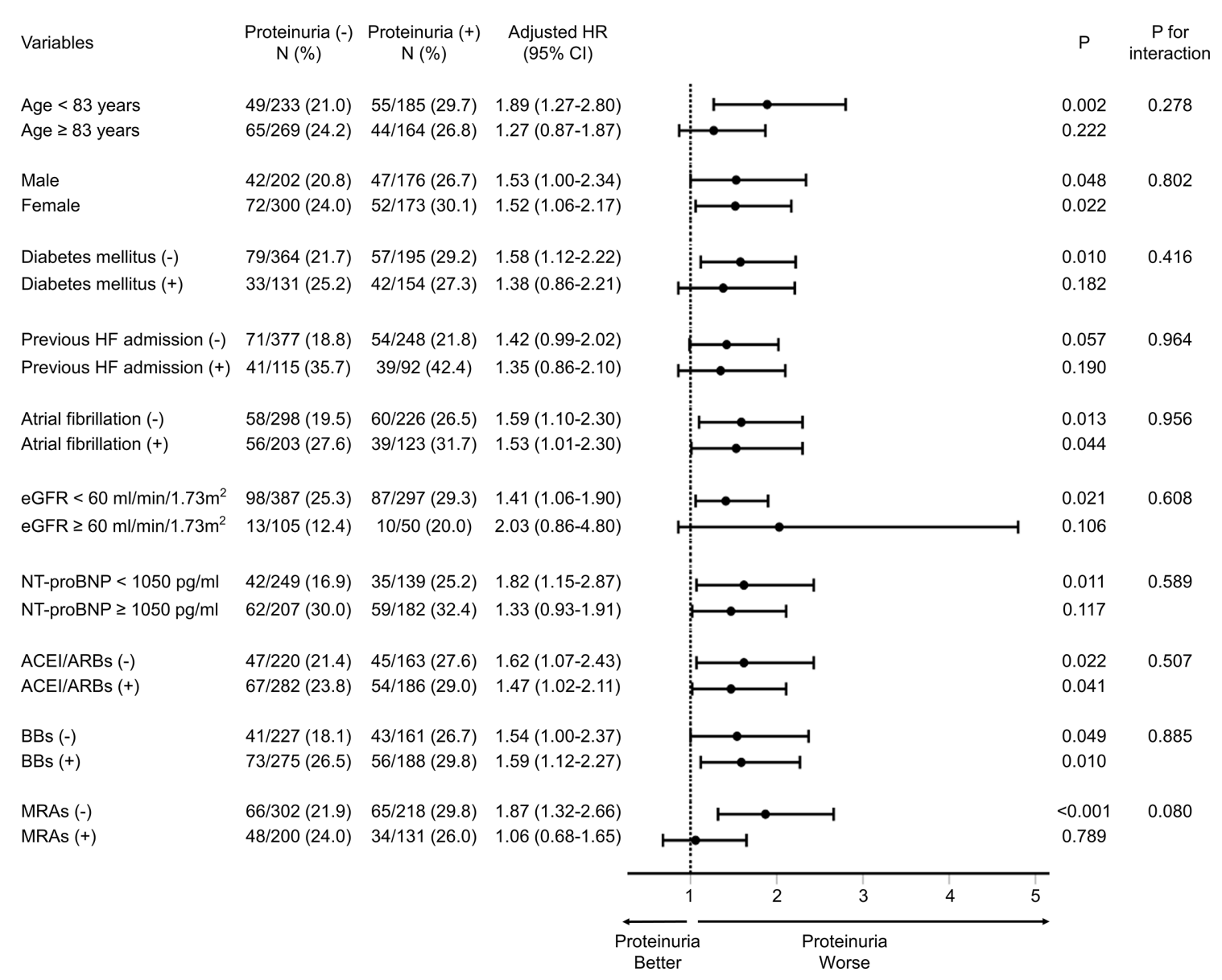

Figure 3 Subgroup analysis to examine the impact of proteinuria on the composite endpoint in different subgroups of patients. HRs were calculated by multivariable Cox regression analysis adjusting for age and female sex. ACEls, angiotensin-converting enzyme inhibitors; ARBs, angiotensin receptor blockers; BBs, $\beta$-blockers; eGFR, estimated glomerular filtration rate; MRAs, mineralocorticoid receptor antagonists; NT-pro-BNP, N-terminal pro B-type natriuretic peptide. Black spots and bar lines indicate adjusted $\mathrm{HRs}$ and their $95 \% \mathrm{Cls}$, respectively. 
other different subgroups of patients, implying a similar prognostic value of proteinuria in these patients for the composite endpoint.

For sensitivity analyses, we constructed the survival curves for the composite endpoint using different cutoff points of proteinuria. Patients with proteinuria $\geq 1+$ were more likely to reach the composite endpoint than those with proteinuria $\leq$ trace, which was similar to the result in figure 2 (online supplemental figure A). When stratifying patients into three groups according to the severity of proteinuria, the composite endpoint showed a linear increase with the severity of proteinuria (log-rank $\mathrm{p}=0.011$ ) (online supplemental figure B).

\section{DISCUSSION}

We found that the presence of proteinuria detected by urine dipstick test was an independent negative prognostic factor in patients with HFpEF. The prognostic significance of proteinuria was independent of apparent renal function, the severity of $\mathrm{HF}$ and other major confounding factors. Sensitive analysis showed that the risk of adverse outcomes increased in a linear manner with the severity of proteinuria. This study is the first to clarify the prognostic impact of sole dipstick proteinuria on clinical outcomes in HFpEF.

\section{Prognostic impact of proteinuria in HFpEF}

An elevated UACR is reportedly associated with an increased risk of cardiovascular events, including allcause death and HF rehospitalisation in patients with diseases such as diabetes, ${ }^{29}$ hypertension ${ }^{30}$ and stable coronary artery disease, ${ }^{31}$ as well as in the general population. ${ }^{32}$ Elevated UACR is a marker of poor prognosis in both HF with reduced ejection fraction(HFrEF) and HFpEF. ${ }^{1126}$ Miura et al $l^{7}$ reported that proteinuria detected with a dipstick test in combination with eGFR was associated with all-cause death in patients with HFpEF. Our current findings are similar to these previous studies, despite the fact that we focused on the cardiac events in our cohort and the identification of proteinuria with a simple dipstick test as a warning sign that should then set off a cascade of testing. However, it should be noted that proteinuria did not have a significant prognostic value for cardiac death in this study, while Miura et $a l^{7}$ found the opposite, although the measurement of eGFR was similarly calculated. This discrepancy may be caused by a smaller sample size with a smaller number of cardiac deaths, and a shorter follow-up period in this study. The older age of our cohort, which may mask the prognostic impact of proteinuria on mortality, may also relate to this discrepancy. Smith et $a l^{16}$ found that CKD evaluated using both eGFR and urine dipstick protein was associated with adverse clinical outcomes in a community-based cohort of patients with $\mathrm{HF}$ with much younger age in a Western country. Racial disparity, different patient background and phenotypes especially between Asian and Western patients with HFpEF such as prevalence of obesity and AF, and disease severity may limit the generalisability of the results found by Smith et al to any elderly HFpEF cohorts like ours, especially in an ageing society. To the best of our knowledge, this is the first study that clarifies the prognostic significance of sole dipstick proteinuria in elderly HFpEF cohort in an East Asian country. Proteinuria detected with a dipstick test was associated with poor prognosis irrespective of renal function, implying the usefulness of this simple urinary analysis in identifying high-risk patients at point of care. Our study confirms and extends the prognostic value of a random dipstick proteinuria test, a cost-effective and simple method at point-of-care settings, especially as a predictor of HF rehospitalisation in patients with $\mathrm{HFpEF}$, while adding knowledge to the existing literature on this topic of interest. This quick urinary dipstick test suffices to be used as a screening test to identify a worse prognosis in HFpEF, allowing selection of these patients to undergo a more extensive workup while sparing patients with normal findings.

\section{Prevalence of proteinuria in $\mathrm{HFpEF}$}

Proteinuria was detected in $41 \%$ of this study cohort, which was higher than in previous studies (approximately $30 \%) .{ }^{716}$ This discrepancy may be due to the fact that this cohort included much older patients with severely reduced eGFR at baseline in addition to a higher prevalence of cardiovascular comorbidities. Moreover, previous studies included cases of both HFrEF and HFpEF, while CKD was reported to be less prevalent in HFrEF. ${ }^{16}$ In contrast, substudies of randomised controlled trials (RCTs) and an observational study of HFpEF found that the proportion of albuminuria assessed by UACR was $25 \%-35 \%$ and $11 \%-14 \%$ for microalbuminuria and macroalbuminuria, respectively, ${ }^{11} 263$ which shared similar findings with our results, despite the different nature of the study cohorts. Therefore, these results suggest that proteinuria is common both in the real-world HFpEF population and in the highly selected populations of RCTs.

\section{Mechanisms underlying the association between proteinuria and prognosis}

Although the mechanisms underlying the association between proteinuria and worse clinical outcomes in HFpEF are not fully understood, possible mechanisms include activation of the renin-angiotensin system (RAS), sympathetic overactivity, oxidative injury and endothelial dysfunction. ${ }^{34}$ These disorders cause progression of cardiac alterations including increased cardiomyocyte stiffness and interstitial fibrosis, ${ }^{35} 36$ which may lead to progression of left ventricular diastolic dysfunction causing pulmonary hypertension, in turn leading to right ventricular dysfunction and ultimately elevation of central venous pressure. Elevated central venous pressure is known to cause renal injury, resulting in proteinuria and deterioration of $\mathrm{GFR},{ }^{37}$ forming the vicious cycle of the cardiorenal syndrome. Activation of RAS, sympathetic overactivity or oxidative injury are all associated with vascular injury with increased vascular resistance 
and stiffness, as well as promoting sodium and water retention. ${ }^{3437}$ Taken together, these mechanisms may be associated with the more frequent rehospitalisations for HF due to increased afterload. In accordance with this hypothesis, patients with proteinuria had a higher systolic blood pressure in this study (table 1).

\section{Clinical implications}

Our findings indicate the prognostic value of proteinuria detected by urine dipstick test in HFpEF. The finding of positive dipstick proteinuria, which is a convenient, low cost and cost-effective method in the screening context, ${ }^{38}$ should alert physicians of the possibility of early kidney damage regardless of eGFR in this population. Appropriate interventions will be needed in this high-risk population. Selvaraj $e t a l^{33}$ reported that spironolactone significantly alleviated proteinuria compared with placebo and that a reduction in albuminuria was independently associated with favourable outcomes. The results of subgroup analysis in this study showed that patients taking MRAs appeared to have a lower risk of the study outcomes than those not taking them, suggesting the potential benefits of this drug in patients with HFpEF presenting with proteinuria. Interestingly, proteinuria had different prognostic meanings between patients with and without MRA use, which was not the case in those with and without ACEI/ARBs. These findings suggest that MRAs, through their more diverse and possibly stronger positive effects than those of ACEI/ARBs on the endothelial dysfunction and oxidative stress that are problematic in patients with HFpEF presenting with proteinuria, ${ }^{20} 33$ may be the reason. RAS blockade is widely known for its therapeutic efficacy in reducing systemic and intraglomerular hydrostatic pressure, which improves proteinuria. ${ }^{39}$ This effect may potentially prevent or delay the progression of kidney dysfunction. Although RAS blockade is reported to simultaneously offer beneficial clinical outcomes in different patient populations, including patients with hypertension, diabetes, CKD and HFrEF, ${ }^{40-43}$ it remains unknown whether similar favourable effects may be observed in patients with HFpEF having proteinuria. Further investigations are warranted to clarify whether interventions including the use of MRAs or RAS inhibitors to reduce or reverse the severity of proteinuria may improve outcomes in this cohort.

\section{Limitations}

Several limitations of this study need to be considered. First, a proteinuria dipstick test may be affected by variations in urine concentration. More concentrated samples may indicate higher levels of proteinuria than may actually be present, while less concentrated samples may give a false-negative or only a trace reading. Second, it should be noticed that the urine dipstick test has low sensitivity and high false-positive rates for the detection of a trace concentration of albuminuria. ${ }^{24}$ On the contrary, the sensitivity analysis in online supplemental figure A may suggest that the frequency of false-positive for the detection of trace proteinuria was not so high, and that patients with 'trace' proteinuria really had proteinuria. In contrast, it is relatively accurate for the detection of an ACR $>300 \mathrm{mg} / \mathrm{g}$ (dipstick $\geq 1+$ ) ${ }^{24}$ Third, given the use of a single dipstick urinalysis, the possibility of missing transient proteinuria cannot be ruled out. Fourth, although the type of urine dipstick test kit may be of interest, we did not have this data. Dipstick kit was left to the discretion of attending physicians and the availability of the kit at each institution. Fifth, we were unable to compare the prognostic utility between dipstick proteinuria and UACR, a well-established prognosticator, due to the unavailability of urine albumin data. Sixth, we cannot determine the precise causal relationship between proteinuria and the severity of HFpEF and clinical outcomes due to the nature of this observational study design. Last but not least, patients included in this study were predominantly East Asian patients with HFpEF having highly prevalent cardiovascular comorbidities. Therefore, the results of this study should be extrapolated with caution in the context of Western patients with HFpEF. Nonetheless, these findings might be useful in Asian patients with HFpEF living in Western countries. In these medically fragile patients, a dipstick test performed between scheduled urinalyses and other major follow-up examinations may flag patients in need of immediate attention.

\section{CONCLUSION}

This study suggests that dipstick proteinuria may be a prognostic marker in the population predominantly including elderly patients with HFpEF having highly prevalent cardiovascular comorbidities. Evaluation of proteinuria by urine dipstick test may be a simple, practical but useful method for risk stratification in HFpEF even in community-based healthcare settings.

\section{Author affiliations}

${ }^{1}$ Department of Cardiovascular Medicine, Osaka University Graduate School of Medicine, Suita, Japan

${ }^{2}$ Department of Social and Environmental Medicine, Osaka University Graduate School of Medicine, Suita, Japan

${ }^{3}$ Division of Cardiology, Amagasaki Chuo Hospital, Amagasaki, Japan ${ }^{4}$ Department of Medical Informatics, Osaka University Graduate School of Medicine, Suita, Japan

${ }^{5}$ Division of Cardiology, Kawanishi City Hospital, Kawanishi, Japan

${ }^{6}$ Division of Cardiology, Osaka Police Hospital, Osaka, Japan

${ }^{7}$ Division of Cardiology, Osaka Rosai Hospital, Sakai, Japan

${ }^{8}$ Division of Cardiology, Osaka General Medical Center, Osaka, Japan

Acknowledgements The authors thank Sugako Mitsuoka, Masako Terui, Nagisa Yoshioka, Satomi Kishimoto, Kyoko Tatsumi and Noriko Murakami for their excellent assistance in data collection, data management and secretarial work. We thank Libby Cone, MD, MA, from DMC Corp. (www.dmed.co.jp <http://www.dmed.co.jp/>) for editing drafts of this manuscript.

Collaborators The OCVC-Heart Failure Investigators; Shunsuke Tamaki, Tetsuya Watanabe, Takahisa Yamada, Takaharu Hayashi, Yoshiharu Higuchi, Masaharu Masuda, Mitsutoshi Asai, Toshiaki Mano, Hisakazu Fuji, Daisaku Masuda, Yoshihiro Takeda, Yoshiyuki Nagai, Shizuya Yamashita, Masami Sairyo, Yusuke Nakagawa, Shuichi Nozaki, Haruhiko Abe, Yasunori Ueda, Masaaki Uematsu, Yukihiro Koretsune, Kunihiko Nagai, Masamichi Yano, Masami Nishino, Jun Tanouchi, Yoh Arita, Shinji Hasegawa, Takamaru Ishizu, Minoru Ichikawa, Yuzuru Takano, Eisai Rin, Yukinori 
Shinoda, Shiro Hoshida, Masahiro Izumi, Hiroyoshi Yamamoto, Hiroyasu Kato, Kazuhiro Nakatani, Yuji Yasuga, Mayu Nishio, Keiji Hirooka, Takahiro Yoshimura, Yoshinori Yasuoka, Akihiro Tani, Yasushi Okumoto, Hideharu Akagi, Yasunaka Makino, Toshinari Onishi, Katsuomi Iwakura, Nagahiro Nishikawa, Yoshiyuki Kijima, Takashi Kitao, Hideyuki Kanai, Wataru Shioyama, Masashi Fujita, Koichiro Harada, Masahiro Kumada, Osamu Nakagawa, Ryo Araki, Takayuki Yamada, Akito Nakagawa, Yoshio Yasumura, Taiki Sato, Akihiro Sunaga, Bolrathanak Oeun, Hirota Kida, Takayuki Kojima, Yohei Sotomi, Tomoharu Dohi, Kei Nakamoto, Katsuki Okada, Fusako Sera, Shinichiro Suna, Hidetaka Kioka, Tomohito Ohtani, Toshihiro Takeda, Daisaku Nakatani, Hiroya Mizuno, Shungo Hikoso, Yasushi Matsumura, Yasushi Sakata.

Contributors B0: conceptualisation, methodology, formal analysis, investigation, writing-original draft, visualisation; SH: conceptualisation, methodology, investigation, writing-original draft, supervision, project administration; DN, SS, TK and YS: methodology, investigation, supervision, writing-review and editing; HM, AN, YN, MY, ST, YY and TY: supervision, investigation, writing-review and editing; KO and TD: supervision, writing-review \& editing; HK and TS: methodology, writingreview and editing; AS: methodology, investigation, validation, writing-review \& editing; and YS: supervision, project administration, investigation, writing-review and editing.

Funding This study was supported by grants from Japan Society for the Promotion of Science KAKENHI (No. JP 17K09496) and Japan Agency for Medical Research and Development (No. JP16lk1010013) and was funded by Roche Diagnostics K.K. and Fuji Film Toyama Chemical, Co. Ltd.

Competing interests The authors declare the following financial interests/ personal relationships which may be considered as potential competing interests: SH reports a relationship with Daiichi Sankyo Company, Bayer, Astellas Pharma, Pfizer Pharmaceuticals and Boehringer Ingelheim Japan that includes: speaking and lecture fees. SH reports a relationship with Roche Diagnostics, FUJIFILM Toyama Chemical and Actelion Pharmaceuticals that includes: funding grants. ND reports a relationship with Roche Diagnostics that includes: speaking and lecture fees. HM reports a relationship with Daiichi Sankyo Company, Kowa Company, Bayer and Pfizer Pharmaceuticals that includes: speaking and lecture fees. HM reports a relationship with Terumo that includes: funding grants. YS reports a relationship with Abbott Vascular Japan, Boston Scientific Japan, TERUMO, Japan Lifeline, Biosensors, Medtronic, Daiichi-Sankyo, Bayer, Boehringer Ingelheim, and Bristol-Myers Squibb that includes: funding grants and speaking and lecture fees. YS reports a relationship with TERUM0, Asahi Intecc, NIPR0, and Shimadzu Corporation that includes: consulting or advisory. YS reports a relationship with Otsuka Pharmaceutical, Ono Pharmaceutical, Daiichi Sankyo Company, Mitsubishi Tanabe Pharma Corporation and Actelion Pharmaceuticals that includes: speaking and lecture fees. YS reports a relationship with Roche Diagnostic, FUJIFILM Toyama Chemical, Abbott Medical Japan, Otsuka Pharmaceutical, Daiichi Sankyo Company, Mitsubishi Tanabe Pharma Corporation and Biotronik that includes: funding grants. YS reports financial support was provided by Japan Society for the Promotion of Science. YS reports financial support was provided by Japan Agency for Medical Research and Development.

\section{Patient consent for publication Not required.}

Ethics approval All participants were thoroughly explained about the study process during the index hospitalization. Written informed consent was obtained from each participant. The study protocol was approved by the Institutional Review Board of all participating facilities, namely Kansai Rosai Hospital Institutional Review Board (approval ID: 16co10g); Kawachi General Hospital Ethics Committee (approval ID was not available, but approved on 26 April 2016); Osaka Rosai Hospital Ethics Committee(approval ID: 28-5); Higashiosaka City Medical Center Institutional Review Board (approval ID: 02-0313); Osaka Prefectural Hospital Organization 0saka General Medical Center Institutional Review Board(approval ID: 28-2002); Hyogo Prefectural Nishinomiya Hospital Ethics Committee (approval ID: H28-3); Ikeda Municipal Hospital Ethics Committee (approval ID: 3280) and Kawanishi City Hospital Institutional Review Board (approval ID: 28001); Rinku General Medical Center Ethics Committee (approval ID: 27-40); Saiseikai Senri Hospital Ethics Committee (approval ID: 280304); Yao Municipal Hospital Institutional Review Board (approval ID: H28-6); Kawasaki Hospital Ethics Committee (approval ID was not available, but approved on 12 May 2016); Minoh City Hospital Ethics Committee (approval ID was not available, but approved on 24 May 2016); National Hospital Organization Osaka National Hospital Second Institutional Review Board (approval ID: 16024); Kano General Hospital Ethics Committee (approval ID was not available, but approved on 9 June 2016); Toyonaka Municipal Hospital Ethics Committee (approval ID: 2 April 2016); Kinan Hospital Ethics Committee 121; Japan Community Health Care Organization Osaka Hospital
Ethics Committee (approval ID: 2016-2); Kobe Ekisaikai Hospital Ethics Committee (approval ID: 2016-3); Sakurabashi Watanabe Hospital Ethics Committee (approval ID: 16-15); Sumitomo Hospital Research Ethics Committee (approval ID: 28-01); Suita Municipal Hospital Institutional Review Board (approval ID: 2017-8); Kinki Central Hospital Ethics Committee (approval ID: 288); Osaka Police Hospital Institutional Review Board (approval ID: 593); Japan Community Health Care Organization Hoshigaoka Medical Center Institutional Review Board (approval ID: 1618); National Hospital Organization Osaka Minami Medical Center Institutional Review Board (approval ID: 28-3); Japan Community Health Care Organization Osaka Minato Central Hospital Ethics Committee (approval ID was not available, but approved on 10 June 2016); Amagasaki Chuo Hospital Ethics Committee (approval ID was not available, but approved on 1 August 2017); Otemae Hospital Institutional Review Board (approval ID: 2017-020); Osaka University Hospital Clinical Research Review Committee (approval ID: 15471); Osaka International Cancer Institute Institutional Review Board (№. 20097).

Provenance and peer review Not commissioned; externally peer reviewed.

Data availability statement All data relevant to the study are included in the article or uploaded as supplementary information. Data sharing is not applicable in this study due to the restrictions of institutional review boards.

Supplemental material This content has been supplied by the author(s). It has not been vetted by BMJ Publishing Group Limited (BMJ) and may not have been peer-reviewed. Any opinions or recommendations discussed are solely those of the author(s) and are not endorsed by BMJ. BMJ disclaims all liability and responsibility arising from any reliance placed on the content. Where the content includes any translated material, BMJ does not warrant the accuracy and reliability of the translations (including but not limited to local regulations, clinical guidelines, terminology, drug names and drug dosages), and is not responsible for any error and/or omissions arising from translation and adaptation or otherwise.

Open access This is an open access article distributed in accordance with the Creative Commons Attribution Non Commercial (CC BY-NC 4.0) license, which permits others to distribute, remix, adapt, build upon this work non-commercially, and license their derivative works on different terms, provided the original work is properly cited, appropriate credit is given, any changes made indicated, and the use is non-commercial. See: http://creativecommons.org/licenses/by-nc/4.0/.

\section{ORCID iDs}

Shungo Hikoso http://orcid.org/0000-0003-2284-1970

Yohei Sotomi http://orcid.org/0000-0002-7564-2978

Yasushi Sakata http://orcid.org/0000-0002-5618-4721

\section{REFERENCES}

1 Lund LH, Savarese G. Global public health burden of heart failure. Cardiac Failure Review 2017;03.

2 Shimokawa H, Miura M, Nochioka K, et al. Heart failure as a general pandemic in Asia. Eur J Heart Fail 2015;17:884-92.

3 Ponikowski P, Voors AA, Anker SD. 2016 ESC guidelines for the diagnosis and treatment of acute and chronic heart failure: the task force for the diagnosis and treatment of acute and chronic heart failure of the european society of cardiology (ESC) developed with the special contribution of the heart failure association (HFA) of the ESC. Eur Heart J 2016;37:2129-200.

4 Yancy CW, Jessup M, Bozkurt B, et al. 2017 ACC/AHA/HFSA focused update of the 2013 ACCF/AHA guideline for the management of heart failure: a report of the american college of cardiology/american heart association task force on clinical practice guidelines and the heart failure society of america. Circulation 2017;136:e137-61.

5 Gori M, Senni M, Gupta DK, et al. Association between renal function and cardiovascular structure and function in heart failure with preserved ejection fraction. Eur Heart J 2014;35:3442-51.

6 Damman K, Valente MAE, Voors AA, et al. Renal impairment, worsening renal function, and outcome in patients with heart failure: an updated meta-analysis. Eur Heart J 2014;35:455-69.

7 Miura M, Shiba N, Nochioka K, et al. Urinary albumin excretion in heart failure with preserved ejection fraction: an interim analysis of the chart 2 study. Eur J Heart Fail 2012;14:367-76.

8 van de Wouw J, Broekhuizen M, Sorop O, et al. Chronic kidney disease as a risk factor for heart failure with preserved ejection fraction: a focus on microcirculatory factors and therapeutic targets. Front Physiol 2019;10:1108.

9 Unger ED, Dubin RF, Deo R, et al. Association of chronic kidney disease with abnormal cardiac mechanics and adverse outcomes in 
patients with heart failure and preserved ejection fraction. Eur $J$ Heart Fail 2016;18:103-12.

10 Huang M-J, Wei R-B, Zhao J, et al. Albuminuria and endothelial dysfunction in patients with non-diabetic chronic kidney disease. Med Sci Monit 2017;23:4447-53.

11 Katz DH, Burns JA, Aguilar FG, et al. Albuminuria is independently associated with cardiac remodeling, abnormal right and left ventricular function, and worse outcomes in heart failure with preserved ejection fraction. JACC Heart Fail 2014;2:586-96.

12 Arai R, Suzuki S, Kano H, et al. Role of dipstick proteinuria for predicting cardiovascular events: a Japanese cardiovascular hospital database analysis. Heart Vessels 2020;35:1256-69.

13 Bailey LN, Levitan EB, Judd SE, et al. Association of urine albumin excretion with incident heart failure hospitalization in communitydwelling adults. JACC Heart Fail 2019;7:394-401.

14 Kojima A, Hanai M, Fukami K. Significance of the measurement and evaluation of the albuminuria in chronic kidney disease patients. Sysmex Journal International 2018;28.

15 Paige NM, Nagami GT. The top 10 things nephrologists WISH every primary care physician knew. Mayo Clin Proc 2009;84:180-6.

16 Smith DH, Thorp ML, Gurwitz JH, et al. Chronic kidney disease and outcomes in heart failure with preserved versus reduced ejection fraction: the cardiovascular research network preserve study. Circ Cardiovasc Qual Outcomes 2013;6:333-42.

17 Suna S, Hikoso S, Yamada T, et al. Study protocol for the PURSUITHFpEF study: a prospective, multicenter, observational study of patients with heart failure with preserved ejection fraction. BMJ Open 2020;10:e038294.

18 Vasan RS, Levy D. Defining diastolic heart failure: a call for standardized diagnostic criteria. Circulation 2000;101:2118-21.

19 Carson P, Massie BM, McKelvie R, et al. The irbesartan in heart failure with preserved systolic function (I-PRESERVE) trial: rationale and design. J Card Fail 2005;11:576-85.

20 Desai AS, Lewis EF, Li R, et al. Rationale and design of the treatment of preserved cardiac function heart failure with an aldosterone antagonist trial: a randomized, controlled study of spironolactone in patients with symptomatic heart failure and preserved ejection fraction. Am Heart J 2011;162:e910:966-72.

21 Solomon SD, Rizkala AR, Gong J, et al. Angiotensin receptor neprilysin inhibition in heart failure with preserved ejection fraction: rationale and design of the PARAGON-HF trial. JACC Heart Fail 2017;5:471-82.

22 Nguyen N-T-V, Tran DT, Le An P. Clinical phenotypes and age-related differences in presentation, treatment, and outcome of heart failure with preserved ejection fraction: a Vietnamese multicenter research. Cardiol Res Pract 2021;2021:1-9.

23 Konta T, Hao Z, Takasaki S, et al. Clinical utility of trace proteinuria for microalbuminuria screening in the general population. Clin Exp Nephrol 2007;11:51-5.

24 Park Jl, Baek H, Kim BR, et al. Comparison of urine dipstick and albumin:creatinine ratio for chronic kidney disease screening: a population-based study. PLoS One 2017;12:e0171106.

25 Sasaki S, Yoshikawa N, Akiba T. Preface: evidence-based practice guideline for the treatment of chronic kidney disease. Clin Exp Nephrol 2009;13:533.
26 Jackson CE, Solomon SD, Gerstein HC, et al. Albuminuria in chronic heart failure: prevalence and prognostic importance. Lancet 2009;374:543-50.

27 Fisher LD, Lin DY. Time-dependent covariates in the COX proportional-hazards regression model. Annu Rev Public Health 1999;20:145-57.

28 Razvi S, Weaver JU, Butler TJ, et al. Levothyroxine treatment of subclinical hypothyroidism, fatal and nonfatal cardiovascular events, and mortality. Arch Intern Med 2012;172:811-7.

29 Gerstein HC, Mann JF, Yi Q, et al. Albuminuria and risk of cardiovascular events, death, and heart failure in diabetic and nondiabetic individuals. JAMA 2001;286:421-6.

30 Wachtell $\mathrm{K}$, Ibsen $\mathrm{H}$, Olsen $\mathrm{MH}$, et al. Albuminuria and cardiovascular risk in hypertensive patients with left ventricular hypertrophy: the life study. Ann Intern Med 2003;139:901-6.

31 Solomon SD, Lin J, Solomon CG, et al. Influence of albuminuria on cardiovascular risk in patients with stable coronary artery disease. Circulation 2007;116:2687-93.

32 van der Velde M, Matsushita K, et al, Chronic Kidney Disease Prognosis Consortium. Association of estimated glomerular filtration rate and albuminuria with all-cause and cardiovascular mortality in general population cohorts: a collaborative meta-analysis. Lancet 2010;375:2073-81.

33 Selvaraj S, Claggett B, Shah SJ, et al. Prognostic value of albuminuria and influence of spironolactone in heart failure with preserved ejection fraction. Circ Heart Fail 2018;11:e005288.

34 Lazzeri C, Valente S, Tarquini R. Cardiorenal syndrome caused by heart failure with preserved ejection fraction. Int $J$ Nephrol 2011;2011:634903-7.

35 Akiyama E, Sugiyama S, Matsuzawa Y, et al. Incremental prognostic significance of peripheral endothelial dysfunction in patients with heart failure with normal left ventricular ejection fraction. J Am Coll Cardiol 2012;60:1778-86.

36 Paulus WJ, Tschöpe C. A novel paradigm for heart failure with preserved ejection fraction: comorbidities drive myocardial dysfunction and remodeling through coronary microvascular endothelial inflammation. J Am Coll Cardiol 2013;62:263-71.

37 Agrawal A, Naranjo M, Kanjanahattakij N, et al. Cardiorenal syndrome in heart failure with preserved ejection fraction-an under-recognized clinical entity. Heart Fail Rev 2019;24:421-37.

38 Kondo M, Yamagata K, Hoshi S-ling, et al. Cost-effectiveness of chronic kidney disease mass screening test in Japan. Clin Exp Nephrol 2012;16:279-91.

39 Lassila M, Cooper ME, Jandeleit-Dahm K. Antiproteinuric effect of Ras blockade: new mechanisms. Curr Hypertens Rep 2004;6:383-92

40 Galle J. Reduction of proteinuria with angiotensin receptor blockers. Nat Clin Pract Cardiovasc Med 2008;5 Suppl 1:S36-43.

41 Oh HJ, Kim CT, Ryu D-R. Effect of renin-angiotensin system blockade on mortality in Korean hypertensive patients with proteinuria. Electrolyte Blood Press 2019;17:25-35.

42 Higuchi S, Kohsaka S, Shiraishi Y, et al. Association of reninangiotensin system inhibitors with long-term outcomes in patients with systolic heart failure and moderate-to-severe kidney function impairment. Eur J Intern Med 2019;62:58-66.

43 Gradman $\mathrm{AH}$. LCZ696: the next step in improving Ras inhibition? Curr Hypertens Rep 2015;17:37. 